\title{
NOTE ON THE LOCATION OF THE CRITICAL POINTS OF A REAL RATIONAL FUNCTION
}

\section{J. L. WALSH}

The object of this note is to study the critical points of a real rational function $p(z)$ of degree $-2 m(>0)$ with $-2 m$ poles in the closed interior of the unit circle $C:|z|=1$, with a real zero of multiplicity $k(>0)$ in the point $x_{1}$ interior to $C$, and with a zero of multiplicity $-2 m-k(>0)$ at infinity. The corresponding problems for the case $\left|x_{1}\right|>1$, and for the case that $p(z)$ is of degree $k$ with infinity no longer a zero but a pole of $p(z)$ of multiplicity $k+2 m(>0)$ with no restrictions on $\left|x_{1}\right|$, have already been treated elsewhere; ${ }^{1}$ we retain the notation and terminology of that previous treatment.

We shall prove

Lemma 5. Let $C:|z|=1$ be the unit circle, let $\lambda(>1)$ be constant, and let $A: z=x_{1}$ be a real point interior to $C$. If $P$ is a variable nonreal point, we denote by $Q$ the intersection other than $P$ of the line $A P$ with the circle through $-1,+1$, and $P$. Then the locus of points $P:(x, y)$ such that we have $Q P / A P=\lambda$ consists of the nonreal points of the circle

$$
(\lambda-1)\left[\left(x-x_{1}\right)^{2}+y^{2}\right]+x_{1}^{2}-1=0 .
$$

If $P$ is the point $(x, y)$, then the circle through $-1,+1$, and $P$ is

$$
X^{2}+\left[Y-\left(x^{2}+y^{2}-1\right) / 2 y\right]^{2}=1+\left(x^{2}+y^{2}-1\right)^{2} / 4 y^{2},
$$

where the running coordinates are $X$ and $Y$. The point $Q$ has the coordinates $\left(\lambda x_{1}+x-\lambda x, y-\lambda y\right)$, and a necessary and sufficient condition that $Q$ lie on the circle (10) is precisely (9). Equation (9) represents a proper circle.

Lemma 6. Let $C:|z|=1$ be the unit circle, and $A: z=x_{1}$ a real point interior to $C$. If the point $P:(x, y)$ lies exterior to the circle (9), then for every point $Q^{\prime}$ collinear with $A$ and $P$, separated by $A$ from $P$, and lying interior to the circle (10), we have $Q^{\prime} P / A P<\lambda$.

It is to be noted that $A$ is the center of the circle (9). When $P$ moves on $A P$ monotonically away from $A$, the point $Q$ of Lemma 5 moves monotonically toward $A$. Consequently the ratio $Q A / A P$

Received by the editors October 23, 1950.

$1 \mathrm{~J}$. L. Walsh, The location of critical points, Amer. Math. Soc. Colloquium Publications, vol. 34, 85.8.3. All references in the present note are to that book. 
$=Q P / A P-1$ decreases monotonically, and Lemma 6 follows.

We are now in a position to establish our main result:

THEOREM 8. Let $p(z)$ be a rational function of degree $-2 m$ all of whose poles lie in the closed interior of the unit circle $C$, which has a zero of multiplicity $k(>0)$ in the real point $x_{1}$ interior to $C$, and which has a zero of multiplicity $-2 m-k(>0)$ at infinity. Then all nonreal finite critical points of $p(z)$ exterior to $C$ lie in the closed interior of the circle

$$
(k+2 m)\left[\left(x-x_{1}\right)^{2}+y^{2}\right]+k\left(1-x_{1}^{2}\right)=0 .
$$

Let $P$ be a nonreal critical point of $p(z)$ exterior to $C$, hence a position of equilibrium in the usual field of force. The particles at the poles of $p(z)$ total $2 m$ in mass, are symmetric in the axis of reals, and lie in the closed interior of $C$; hence, by the method of proof of Lemma 2, the force these particles exert at $P$ is equal to the force exerted at $P$ by a single particle of mass $2 m$ located at some point $Q^{\prime}$ of $R$ (notation of Lemma 2). Then $P$ is a position of equilibrium in the field due to this particle at $Q^{\prime}$, and to a particle of mass $k$ in the point $A: z=x_{1}$. Consequently $Q^{\prime}$ lies in $R_{0}$ (notation of Lemma 2), $P$ is collinear with $A$ and $Q^{\prime}$, and we have $Q^{\prime} P / A P=-2 m / k$. It follows from Lemma 6 with $\lambda=-2 m / k$ that $P$ lies on or within the circle (11). Theorem 8 is established.

From a general result on circular regions ( $\$ 4.2 .4$, Theorem 4 ), it follows that under the hypothesis of Theorem 8 all real critical points of $p(z)$ lie in the interval $S$ :

$$
\left(-2 m x_{1}-k\right) /(-2 m-k) \leqq z \leqq\left(-2 m x_{1}+k\right) /(-2 m-k) .
$$

In order to study the actual locus of critical points in Theorem 8 , we consider a somewhat more general situation:

THEOREM 9. Let a region $R$ symmetric in the axis of reals be the locus of $q(>4)$ poles of a real rational function $p(z)$. Then each point of $R$ belongs to the locus of critical points of $p(z)$.

As in the proof of $\$ 4.2 .2$, Theorem 1 , we find it convenient to consider $R(z) \equiv 1 / p(z)$, and shall prove that each (interior) point of $R$ can be a critical point not a multiple zero of $R(z)$. This conclusion is valid for a real point $\alpha_{0}$ of $R$, for let $\alpha_{0}$ lie interior to $R$ on the axis of reals; precisely the method of $\$ 4.2 .2$ then shows that $\alpha_{0}$ can be a critical point not a multiple zero of $R(z)$.

Let now $\alpha_{0}$ be a nonreal point of $R$; we set

$$
R(z) \equiv\left(z-\alpha_{0}\right)\left(z-\bar{\alpha}_{0}\right)(z-\alpha)(z-\bar{\alpha}) R_{1}(z),
$$


where $\alpha$ is allowed to vary in the neighborhood of the fixed point $\alpha_{0}$, but where the zeros and poles of $R_{1}(z)$ are fixed and remote from $\alpha_{0}$. The critical points of $R(z)$ are the zeros of $R^{\prime}(z) \equiv\left(z-\bar{\alpha}_{0}\right)(z-\alpha)$ $\cdot(z-\bar{\alpha}) R_{1}(z)+\left(z-\alpha_{0}\right)(z-\alpha)(z-\bar{\alpha}) R_{1}(z)+\left(z-\alpha_{0}\right)\left(z-\bar{\alpha}_{0}\right)(z-\bar{\alpha}) R_{1}(z)$ $+\left(z-\alpha_{0}\right)\left(z-\bar{\alpha}_{0}\right)(z-\alpha) R_{1}(z)+\left(z-\alpha_{0}\right)\left(z-\bar{\alpha}_{0}\right)(z-\alpha)(z-\bar{\alpha}) R_{1}^{\prime}(z)=0$. This equation defines $z$ as an implicit function of $\alpha$, and the equation is satisfied for $z=\alpha=\alpha_{0}$. Even though $z$ is not an analytic function of $\alpha$, we have by differentiation for the values $z=\alpha=\alpha_{0}$

$$
\begin{aligned}
& \frac{\partial R^{\prime}(z)}{\partial z}=2\left(\alpha_{0}-\bar{\alpha}_{0}\right)^{2} R_{1}\left(\alpha_{0}\right), \\
& \frac{\partial R^{\prime}(z)}{\partial \alpha}=-\left(\alpha_{0}-\bar{\alpha}_{0}\right)^{2} R_{1}\left(\alpha_{0}\right) .
\end{aligned}
$$

We interpret $R^{\prime}(z)=0$ as two real equations in four real variables, the real and pure imaginary parts of $z$ and $\alpha$; the jacobian for the values $z=\alpha=\alpha_{0}$ has a value which is different from zero. It follows from the implicit function theorem for real variables that the equation $R^{\prime}(z)=0$ defines $z$ as a function of $\alpha$, and when $\alpha$ varies throughout a neighborhood of $\alpha_{0}$ then $z$ also varies throughout a neighborhood of $\alpha_{0}$; it is readily shown also from the proof of the implicit function theorem based on successive approximations that if $\alpha_{0}$ is now allowed to vary over a small neighborhood, then a neighborhood of $\alpha_{0}$ of fixed size as a locus of $\alpha$ corresponds to a neighborhood of $\alpha_{0}$ as a locus of $z$ which contains uniformly a circle of constant positive radius whose center is the variable $\alpha_{0}$. The proof of Theorem 9 can now be completed as was the proof of $\$ 4.2 .2$, Theorem 1 .

We return now to Theorem 8 . If we replace the problem represented by Theorem 8 by the more general problem of finding the critical points of a function of the form $(0<k<-2 m)$

$$
\begin{array}{r}
p(z) \equiv\left(z-x_{1}\right)^{k} /\left(z-\alpha_{1}\right)^{m_{1}}\left(z-\bar{\alpha}_{1}\right)^{m_{1}}\left(z-\alpha_{2}\right)^{m_{2}}\left(z-\bar{\alpha}_{2}\right)^{m_{2}} \\
\cdots\left(z-\alpha_{n}\right)^{m^{n}}\left(z-\bar{\alpha}_{n}\right)^{m_{n}},
\end{array}
$$

where we have $\left|x_{1}\right|<1,\left|\alpha_{j}\right| \leqq 1, m_{j}>0, m_{1}+m_{2}+\cdots+m_{n}=-m$, and where $k, m, x_{1}$ are given but $k, m_{1}, m_{2}, \cdots, m_{n}, m$ need no longer be integral, then the interior of $C$ plus the closed interior of (11) plus $S$ plus the point at infinity is the precise locus of critical points of $p(z)$. Any point $z$ interior to $C$ can be a critical point of $p(z)$, as follows from Theorem 9. Any point of $S$ can be a critical point of $p(z)$; compare $\$ 4.2 .4$, Theorem 4. Any nonreal point $P$ in the closed interior of (9) not interior to $C$ can be a critical point of $p(z)$, for if $P$ is given there 
exists a point $Q^{\prime}$ on the line $P A$ extended, which is contained in the closed region $R_{0}$ (notation of Lemma 2) and with $Q^{\prime} P / A P=-2 m / k$; it follows from the proof of Lemma 2 that a suitable choice of negative particles in $C$ of total mass $2 m$ is equivalent (so far as concerns the force at $P$ ) to a $(2 m)$-fold negative particle at $Q^{\prime}$; thus $P$ is a critical point of a suitably chosen $p(z)$ of type (12). Every real point interior to (11) lies in $C$ or $S$, and the conclusion of Theorem 8 persists, so the locus of critical points of (12) is as stated.

Under the original conditions of Theorem 8 , with $x_{1}$ given, every real point interior to $C$ belongs to the locus of critical points if we have $-m=1$, and every point interior to $C$ belongs to the locus if we have $-m>1$.

If the hypothesis of Theorem 8 is modified slightly, the conclusion requires large modification:

THEOREM 10. Let $p(z)$ be a rational function of form (12) where we have $\left|x_{1}\right|<1,\left|\alpha_{j}\right| \leqq 1, m_{j}>0, m_{1}+m_{2}+\cdots+m_{n}=-m$, and where $m, x_{1}$ are given, but $k, m_{1}, m_{2}, \cdots, m_{n}, m$ need not be integral. Then the locus of critical points of $p(z)$ for all possible choices of $k, m_{1}, m_{2}, \cdots$, $m_{n}, \alpha_{j}$, consists of the extended plane.

The proof of Theorem 10 is similar to the previous proof of the locus property of (12), and is left to the reader.

HARVARD UNIVERSITY 